\title{
Efficacité De La Pollinisation Entomophile Du Palmier A Huile Dans Les Plantations Du Sud-Ouest Et Du Sud-Est De La Côte d'Ivoire
}

\author{
Kouakou Malanno, \\ Laboratoire de l'unité de recherche d'entomologie agricole \\ Université Nangui Abrogou, Abidjan, Côte d'Ivoire \\ Centre National de Recherche Agronomique (CNRA), Côte d'Ivoire \\ Hala Kinampinan Adelphe, \\ Laboratoire de zoologie et de biologie animale \\ Université Félix Houphouët-Boigny, Abidjan, Côte d'Ivoire

\section{Hala N'klo,} \\ Centre National de Recherche Agronomique (CNRA), Côte d'Ivoire
}

\section{Dagnogo Mamadou,}

Laboratoire de l'unité de recherche d'entomologie agricole

Université Nangui Abrogou, Abidjan, Côte d'Ivoire

Doi: 10.19044/esj.2018.v14n12p392 URL:http://dx.doi.org/10.19044/esj.2018.v14n12p392

\begin{abstract}
Low fruit set rates in oil palm plantations may be related to inadequate entomophilous pollination. The objective of this study was to follow the variations of fruit set and to determine its relationships with the entomofauna visiting inflorescences at La Mé, Grand-Béréby and Iboké in Côte d'Ivoire. Densities of the insects were followed out on male and female inflorescences during 12 months. The fruit set rate was also monitored over the same period on young bunches. The results showed that pollination is provided by Elaeidobius kamerunicus, Elaeidobius plagiatus, Elaeidobius singularis, Elaeidobius subvittatus, Microporum congolense, Microporum dispar and Atheta burgeoni. At La Mé, the fruit set rate was negatively correlated with the damage of Prosœustus spp $(\mathrm{r}=-0.43, \mathrm{p}=0.01)$ and positively with the abundance of $E$. kamerunicus and E. plagiatus on female flowers (respectively: $\mathrm{r}=0.60, \mathrm{p}=0.002$ and $\mathrm{r}=0.47, \mathrm{p}=0.020$ ). At Grand-Béréby, it is negatively correlated with the damage of Prosœustus $\operatorname{spp}(\mathrm{r}=-0.42, \mathrm{p}=$ $0.01)$. At Iboké, positive correlations were observed with the density of $E$. subvittatus $(\mathrm{r}=0.40, \mathrm{p}<0.001)$, M. congolense $(\mathrm{r}=0.41, \mathrm{p}=0.046)$, M. dispar $(\mathrm{r}=0.57, \mathrm{p}=0.004)$ and A. burgeoni $(\mathrm{r}=0.65, \mathrm{p}<0.001)$. This study highlighted the necessity to manage the populations of pollinators and the
\end{abstract}


inflorescences pest insects to improve the production of oil palm in Côte d'Ivoire.

Keywords: Pollinating insects, Oil palm, Fruit set, Côte-d'Ivoire

\section{Résumé}

Les faibles taux de nouaison des fruits dans les plantations de palmiers à huile peuvent être liés à une insuffisance de la pollinisation entomophile. L'objectif de cette étude était de suivre les variations de la nouaison et de déterminer ses relations avec l'entomofaune visitant les inflorescences à La Mé, Grand-Béréby et Iboké en Côte d'Ivoire. Les densités des insectes ont été suivies sur les inflorescences mâles et femelles pendant 12 mois. Le taux de nouaison a également été suivi sur la même période sur des jeunes régimes. Les résultats ont montré que la pollinisation est assurée par Elaeidobius kamerunicus, Elaeidobius plagiatus, Elaeidobius singularis, Elaeidobius subvittatus, Microporum congolense, Microporum dispar et Atheta burgeoni. A La Mé, le taux de nouaison est corrélé négativement avec les dégâts de Proscustus spp $(\mathrm{r}=-0,43, \mathrm{p}=0,01)$ et positivement avec le nombre de $E$. kamerunicus et $E$. plagiatus visitant les fleurs femelles (respectivement: $\mathrm{r}=$ $0,60, p=0,002$ et $\mathrm{r}=0,47, \mathrm{p}=0,020)$. À Grand-Béréby, il est corrélé négativement avec les dégâts de Prosœustus $\operatorname{spp}(\mathrm{r}=-0,42, \mathrm{p}=0,01)$. A Iboké, des corrélations positives ont été observées avec la densité de E. subvittatus (r $=0,40, \mathrm{p}<0,001), M$. congolense $(\mathrm{r}=0,41, \mathrm{p}=0,046)$, M. dispar $(\mathrm{r}=0,57, \mathrm{p}$ $=0,004)$ et $A$. burgeoni $(\mathrm{r}=0,65, \mathrm{p}<0,001)$. Cette étude a mis en évidence la nécessité de gérer les populations de pollinisateurs et les insectes ravageurs des inflorescences pour améliorer la production du palmier à huile en Côte d'Ivoire.

Mots clés : Insectes pollinisateurs, Palmier à huile, taux de nouaison, Côted'Ivoire

\section{Introduction}

La pollinisation du palmier à huile est essentiellement réalisée par des insectes (Syed, 1979 ; Appiah, 1999 ; Appiah et Agyei-Dwarko, 2013). L'absence de certains de ces insectes pollinisateurs ou la diminution de leurs populations dans les palmeraies entraîne systématiquement une diminution du taux de fruits fécondés (Syed et al., 1982 ; Mariau et Genty, 1988; Mariau et al., 1991 ; Appiah et Agyei-Dwarko, 2013 ; Agus et al., 2014).

En Côte d'Ivoire, près de vingt (20) espèces d'insectes sont inféodées aux inflorescences du palmier à huile. Parmi ces espèces, quatre Coléoptères Curculionidés du genre Elaeidobius assurent l'essentiel de la pollinisation. Il s'agit de E. kamerunicus Faust, E. plagiatus Faust, E. subvittatus Faust et E. 
singularis Faust (Syed, 1979 ; Desmier de Chenon, 1981, Mariau et Genty, 1988; Mariau et al., 1991). L'espèce E. kamerunicus transporte plus de grains de pollen et s'est montré plus efficace dans la pollinisation (Kouakou et al., 2014)

Les travaux récents sur l'entomofaune des inflorescences du palmier à huile en Côte d'Ivoire ont révélé des fluctuations spatio-temporelles importantes et une diminution de la densité de ces principaux pollinisateurs (Hala et al., 2012 ; Koua et al., 2013). L'influence de ces fluctuations sur le taux de nouaison n'a pas été clairement établie. Or, c'est ce paramètre qui permet d'apprécier la qualité de la pollinisation. Par ailleurs, ces travaux se sont déroulés sur la station de La Mé, située au sud-est du pays. Il serait intéressant de disposer de données sur le sud-ouest qui constitue, à l'heure actuelle, la principale zone d'expansion de cette culture. Cette étude a eu pour objectif d'établir les relations entre les différents insectes fréquentant les inflorescences du palmier à huile et le taux de nouaison dans les localités du sud-est et du sud-ouest de la Côte d'Ivoire.

\section{Matériel et méthodes}

\section{Sites d'étude}

L'étude a été conduite sur trois sites : les plantations de la station de recherche de La Mé, appartenant au Centre National de Recherche Agronomique (CNRA) situées au sud-est (Latitude : $5^{\circ} 26^{\prime} \mathrm{N}$ et Longitude : $\left.3^{\circ} 50^{\prime} \mathrm{W}\right)$, les plantations de la Société des Caoutchoucs de Grand-Béréby (SOGB) situées à Grand-Béréby au sud-ouest (Latitude : $5^{\circ} 0^{\prime} \mathrm{N}$ et Longitude $6^{\circ} 5^{\prime} \mathrm{W}$ ) et les plantations de la Société des Palmes de Côte-d'Ivoire (PALM$\mathrm{CI})$ situées à Iboké au sud-ouest (Latitude : $4^{\circ} 8^{\prime} \mathrm{N}$ et Longitude : $7^{\circ} 4^{\prime} \mathrm{W}$ ) (Figure 1).

Ces localités sont situées dans la zone forestière du pays. Le climat y est de type subéquatorial, chaud et humide, caractérisé par deux saisons pluvieuses et deux saisons sèches. La première saison sèche s'étend de décembre à avril et la seconde saison sèche, d'août à septembre. La première saison des pluies intervient de mai à juillet, et la seconde, d'octobre à novembre. La pluviométrie moyenne annuelle peut atteindre 1400 à 2400 $\mathrm{mm}$. Cette zone bénéficie d'une humidité relative assez importante (80 à $90 \%$ ) et d'une température plus ou moins constante $\left(28\right.$ à $\left.29^{\circ} \mathrm{C}\right)$ (Brou, 2010). 


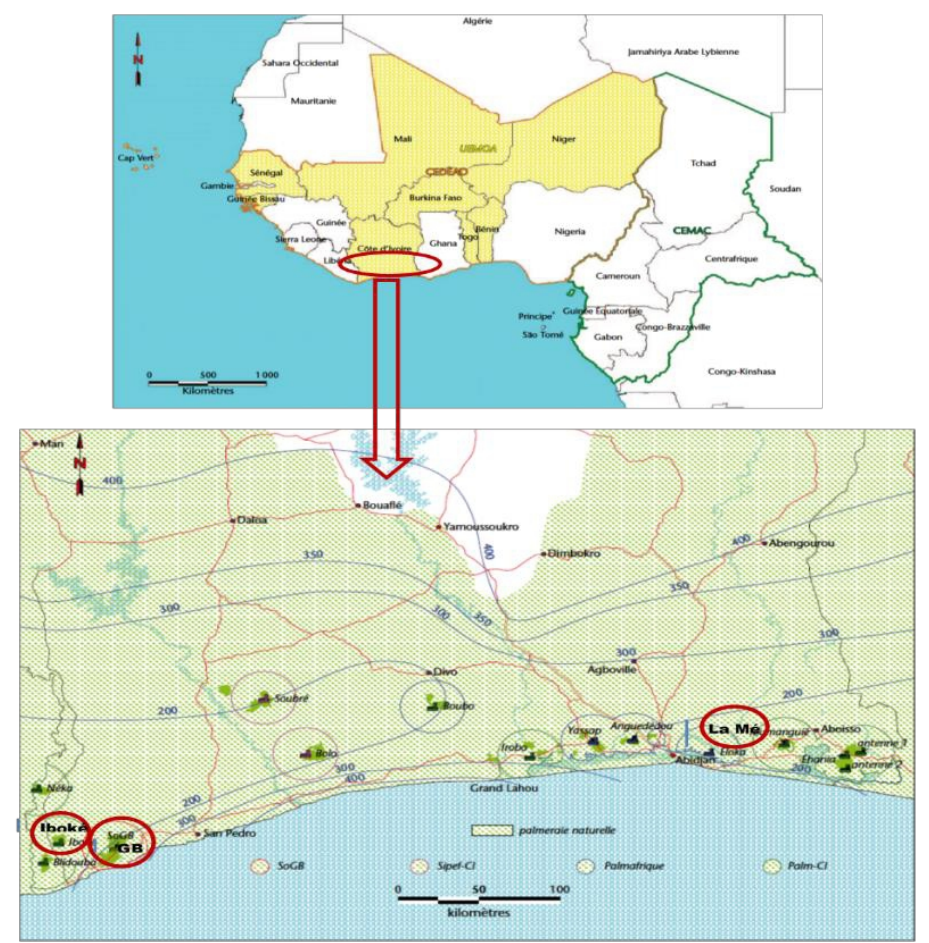

Figure 5 : Localisation des sites de La Mé, SOGB (Grand-Béréby) et PALM-CI Iboké (Jannot, 2010)

\section{Dispositif d'étude}

Sur chaque site d'étude, trois plantations ont été retenues (Tableau 1). Dans chaque plantation, une parcelle d'observation comportant 20 lignes de 10 arbres a été délimitée et les arbres ont été numérotés pour faciliter leur repérage.

Tableau 1 : Caractéristiques des parcelles d'étude selon les sites de production

\begin{tabular}{|c|c|c|c|c|c|c|}
\hline Sites & Parcelle & $\begin{array}{l}\text { Coordonnées } \\
\text { géographiques }\end{array}$ & Zone & $\begin{array}{l}\text { Année de } \\
\text { plantation }\end{array}$ & $\begin{array}{l}\text { Superficie } \\
\text { (ha) }\end{array}$ & $\begin{array}{l}\text { Variété } \\
\text { cultivée }\end{array}$ \\
\hline \multirow{3}{*}{$\begin{array}{c}\text { Station CNRA } \\
\text { / La Mé }\end{array}$} & PI-H65 & $5^{\circ} 28^{\prime} \mathrm{N} 3^{\circ} 52^{\prime} \mathrm{W}$ & PL & 2005 & 6,67 & $\mathrm{C} 1001 \mathrm{~F}$ \\
\hline & PI-F12 & $5^{\circ} 26^{\prime} \mathrm{N} 3^{\circ} 50^{\prime} \mathrm{W}$ & PL & 2006 & 2,0 & $\mathrm{C} 1001 \mathrm{~F}$ \\
\hline & PI-J31 & $5^{\circ} 27^{\prime} \mathrm{N} 3^{\circ} 50^{\prime} \mathrm{W}$ & PL & 2007 & 3,31 & $\mathrm{C} 1001 \mathrm{~F}$ \\
\hline \multirow{3}{*}{$\begin{array}{l}\text { Zone SOGB / } \\
\text { Grand-Béréby }\end{array}$} & PI-BP05 & $4^{\circ} 40^{\prime} \mathrm{N} 7^{\circ} 06^{\prime} \mathrm{W}$ & PL & 2001 & 10,87 & C1001F \\
\hline & PI-SP30 & $4^{\circ} 39^{\prime} \mathrm{N} 7^{\circ} 05^{\prime} \mathrm{W}$ & $\mathrm{BF}$ & 2005 & 12,19 & $\mathrm{C} 1001 \mathrm{~F}$ \\
\hline & PV-SS & $4^{\circ} 40^{\prime} \mathrm{N} 7^{\circ} 01^{\prime} \mathrm{W}$ & PL & 2002 & 18,0 & $\mathrm{C} 1001 \mathrm{~F}$ \\
\hline \multirow{3}{*}{$\begin{array}{l}\text { Zone PALMCI } \\
\text { / Iboké }\end{array}$} & PI-C3P4 & $4^{\circ} 44^{\prime} \mathrm{N} 7^{\circ} 21^{\prime} \mathrm{W}$ & $\mathrm{BF}$ & 2003 & 26,63 & $\mathrm{C} 1001 \mathrm{~F}$ \\
\hline & PI-C3P5 & $4^{\circ} 44^{\prime} \mathrm{N} 7^{\circ} 21^{\prime} \mathrm{W}$ & PL & 2003 & 43,30 & $\mathrm{C} 1001 \mathrm{~F}$ \\
\hline & $\begin{array}{c}\text { PV- } \\
\text { DAPO }\end{array}$ & $4^{\circ} 44^{\prime} \mathrm{N} 7^{\circ} 21^{\prime} \mathrm{W}$ & PL & 2002 & 6,0 & $\mathrm{C} 1001 \mathrm{~F}$ \\
\hline
\end{tabular}

$\mathbf{P I}=$ Plantation Industrielle $; \mathbf{P V}=$ Plantation villageoise $; \mathbf{P L}=$ plateau $; \mathbf{B F}=$ Bas-fond. 


\section{Suivi de la densité des insectes pollinisateurs sur les inflorescences Collecte sur les inflorescences femelles}

Chaque mois, quatre inflorescences femelles ont été repérées dans chaque parcelle et protégées avant la floraison, à l'aide de la mousseline. Le jour de la pleine anthèse, ces inflorescences ont été surveillées pour capturer à l'aide de l'aspirateur à bouche, tous les insectes qui arrivent sur la mousseline pendant les intervalles de temps suivants :

- 09 heures à 10 heures : 10 minutes de capture

- 11 heures à 12 heures : 10 minutes de capture

- 16 heures à 17 heures : 10 minutes de capture

Ces horaires ont été retenus sur la base de travaux antérieurs qui ont montré que l'activité de ces insectes est essentiellement diurne. Leur trafic sur les fleurs femelles devient important aux environs de 9 heures, atteignant son pic entre 11 heures et 12 heures. Dans la soirée, le plus grand nombre de visite est observé entre 16 heures et 17 heures (Hala et al., 2012 ; Tuo, 2013 ; Jianjun et al., 2015).

\section{Collecte sur les inflorescences mâles}

Tous les mois, le jour de la collecte sur les inflorescences femelles, quatre inflorescences mâles en pleine anthèse ont été repérées et choisies au hasard dans chaque répétition. Sur chacune d'elles, quatre épillets ont été prélevés délicatement à l'aide d'un sécateur pour être introduits dans un sachet plastique. Les insectes vivant sur ces épillets ont ensuite été neutralisés à l'aide d'un insecticide avant d'être recueillis dans des piluliers étiquetés et contenant de 1 'alcool à $70 \%$. Ces collectes ont été réalisées avant 9 heures du matin, où les insectes sont moins actifs. Les données ont servi à la détermination de la densité des différentes espèces d'insectes sur les fleurs mâles, c'est-à-dire le nombre d'individus par épillet.

\section{Identification des espèces d'insectes}

Les insectes collectés sur les deux types d'inflorescences ont été identifiés jusqu'au niveau de l'espèce sur la station de La Mé. Cette identification a été faite à l'aide d'une loupe binoculaire en se servant de la collection des insectes des inflorescences du palmier à huile disponible sur cette station. Les travaux antérieurs ont également été consultés pour cette identification (Hala et al., 2012).

\section{Suivi du taux de fruits noués et de fruits détruits par les ravageurs}

Le taux de nouaison a été suivi au cours de la période d'étude, en adoptant la méthode décrite par Lecoustre et De Reffey (1987) et Mariau et al. (1991).

Chaque mois, le jour de la collecte des insectes, huit (8) inflorescences 
femelles en pleine floraison, c'est-à-dire en pleine attraction, ont été choisies au hasard et marquées. Un mois après la fécondation, les jeunes régimes issus de ces inflorescences ont été récoltés et les épis ont été séparés de la rafle à l'aide d'une hachette. Sur chaque régime, 50 épis ont été tirés au hasard. Sur ces épis, les fruits noués (ou fécondés) ont été dénombrés.

Sur ces mêmes régimes, les fruits avortés par les attaques de Prosœustus minor et Prosœustus sculptilis (Coleoptera: Curculionidae), principaux ravageurs des inflorescences femelles, ont également été déterminés (Philippe, 1993).

\section{Analyse des données}

Les analyses statistiques de l'étude ont été réalisées à l'aide du logiciel IBM SPSS Statistics 20. Pour évaluer l'impact des insectes pollinisateurs et l'incidence des attaques de ravageurs sur le taux de nouaison, il a été réalisé des analyses de corrélation.

\section{Résultats}

\section{Abondance des différences espèces d'insectes sur les inflorescences}

La composition en espèces de l'entomofaune des inflorescences mâles a été semblable dans les trois localités. Au total, 17 espèces d'insectes ont été observées dans chacune des localités d'étude (Tableaux II, III et IV). Dix espèces fréquentent les deux types d'inflorescences. Il s'agit de cinq Coleoptera Curculionidae du genre Elaeidobius (E. kamerunicus Faust, E. plagiatus Faust, E. singularis Faust, E. subvittatus Faust et E. bilineatus Faust), de deux Coleoptera Curculionidea du genre Prosœustus (P. minor Mshl et $P$. sculptilis Faust), de deux Coleoptera Nitidulidae du genre Microporum (M. congolense Grouvelle et $M$. dispar Watehouse) et du Coleoptera Staphilinidae Atheta burgeoni Bernhauer.

A. burgeoni représente environ $50 \%$ des insectes collectés sur les fleurs mâles (48 à $52 \%$ selon les parcelles), suivie par l'espèce $E$. subvittatus, avec environ 27 à $30 \%$. Sur les fleurs femelles par contre, l'espèce $E$. subvittatus a été la plus abondante (30 à $47 \%$ ). Elle est suivie à La Mé par $A$. burgeoni (13\%) et E. kamerunicus (11\%), à Grand-Béréby par P. sculptilis $(10,51 \%)$ et $M$. congolense $(10,51 \%)$ et à Iboké par $M$. congolense $(17,57$ $\%)$, A. burgeoni (13,46\%) et P. sculptilis (10,13\%) (Tableaux 2, 3 et 4).

Tableau 2 : Abondance des différentes espèces d'insectes sur les inflorescences mâles et femelles du palmier à huile à La Mé

\begin{tabular}{lllll} 
& \multicolumn{2}{l}{ Inflorescences mâles } & \multicolumn{2}{l}{ Inflorescences femelles } \\
\hline Espèces & Nombre & $\%$ & Nombre & $\%$ \\
\hline Elaeidobius kamerunicus (Faust) & 6069 & 1,97 & 2151 & 11,19 \\
Elaeidobius plagiatus (Faust) & 32588 & 8,98 & 493 & 2,08 \\
Elaeidobius singularis (Faust) & 13814 & 3,73 & 767 & 4,15
\end{tabular}




\begin{tabular}{lllll} 
Elaeidobius subvittatus (Faust) & 139983 & 26,93 & 9192 & 45,01 \\
Elaeidobius bilineatus (Faust) & 2878 & 0,57 & 487 & 2,33 \\
Prosœustus sculptilis (Faust) & 182 & 0,07 & 1095 & 7,17 \\
Prosœustus minor (Mshl) & 43 & 0,01 & 1174 & 7,6 \\
Microporum congolense & 5956 & 1,45 & 654 & 4,72 \\
(Grouvelle) & 11666 & 2,72 & 374 & 3,03 \\
Microporum dispar (Watehouse) & 252807 & 50,49 & 2106 & 12,72 \\
Atheta burgeoni (Bernhauer) & 130 & 0,04 & - & - \\
Gabrius sp. (Stephens) & 760 & 0,22 & - & - \\
Lithargus sp. (Erickson) & 114 & 0,03 & - & - \\
Anthocoris sp. & 15811 & 2,79 & - & - \\
Thrips sp. & & & & \\
\hline
\end{tabular}

Tableau 3 : Abondance des différentes espèces d'insectes sur les inflorescences mâles et femelles du palmier à huile à Grand-Béréby

\begin{tabular}{lllll} 
& \multicolumn{2}{c}{ Inflorescences mâles } & \multicolumn{2}{c}{ Inflorescences femelles } \\
\hline Espèces & Nombre & $\%$ & Nombre & $\%$ \\
\hline Elaeidobius kamerunicus (Faust) & 6550 & 2,15 & 1082 & 9,96 \\
Elaeidobius plagiatus (Faust) & 12542 & 4,12 & 190 & 1,86 \\
Elaeidobius singularis (Faust) & 9066 & 2,98 & 882 & 6,8 \\
Elaeidobius subvittatus (Faust) & 93019 & 30,56 & 5486 & 47,55 \\
Elaeidobius bilineatus (Faust) & 1337 & 0,44 & 383 & 3,11 \\
Prosœustus sculptilis (Faust) & 71 & 0,02 & 1183 & 10,51 \\
Prosœustus minor (Mshl) & 337 & 0,11 & 410 & 5,22 \\
Microporum congolense (Grouvelle) & 4044 & 1,33 & 1183 & 10,51 \\
Microporum dispar (Watehouse) & 9388 & 3,08 & 410 & 5,22 \\
Atheta burgeoni (Bernhauer) & 157835 & 51,86 & 1196 & 8,34 \\
Gabrius sp. (Stephens) & 337 & 0,11 & - & - \\
Lithargus sp. (Erickson) & 436 & 0,14 & - & - \\
Anthocoris sp. & 37 & 0,01 & - & - \\
Thrips sp. & 9333 & 3,07 & - & - \\
\hline
\end{tabular}

Tableau 4 : Abondance des différentes espèces d'insectes sur les inflorescences mâles et femelles du palmier à huile à Grand-Béréby

\begin{tabular}{lllll} 
& \multicolumn{2}{l}{ Inflorescences mâles } & \multicolumn{2}{l}{ Inflorescences femelles } \\
\hline Espèces & Nombre & $\%$ & Nombre & $\%$ \\
\hline Elaeidobius kamerunicus (Faust) & 9192 & 3,46 & 963 & 7,71 \\
Elaeidobius plagiatus (Faust) & 18296 & 6,89 & 310 & 3,59 \\
Elaeidobius singularis (Faust) & 10630 & 4,01 & 761 & 6,43 \\
Elaeidobius subvittatus (Faust) & 71052 & 26,77 & 3014 & 31,55 \\
Elaeidobius bilineatus (Faust) & 1001 & 0,38 & 127 & 1,06 \\
Prosæustus sculptilis (Faust) & 338 & 0,13 & 842 & 10,13 \\
Prosæustus minor (Mshl) & 4114 & 1,55 & 233 & 4,83 \\
Microporum congolense & 6976 & 2,63 & 1395 & 17,57
\end{tabular}


(Grouvelle)

Microporum dispar (Watehouse)

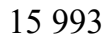

6,03

329

3,67

Atheta burgeoni (Bernhauer)

127184

47,92

725

13,46

Gabrius sp. (Stephens)

181

0,07

Lithargus sp. (Erickson)

290

152

0,11

Anthocoris sp.

04

0,06

Thrips sp.

0,00

\section{Taux de nouaison moyen sur les sites de production}

La Figure 2 présente le pourcentage de fruits fécondés (taux de nouaison) par site. Le taux de nouaison à La Mé a été d'environ 51,21 $12 \%$. A Grand-Béréby, ce taux a été de 52,82 $\pm 15 \%$. Enfin, à Iboké, la proportion de fruits noués par régime a été d'environ $58,63 \pm 12 \%$. Il est ressorti des analyses que le taux de nouaison moyen est significativement plus élevé à Iboké $(\mathrm{ddl}=215, F=6,21, \mathrm{p}=0,002)$. Les moyennes observées à La Mé et à Grand-Béréby ne diffèrent pas significativement.

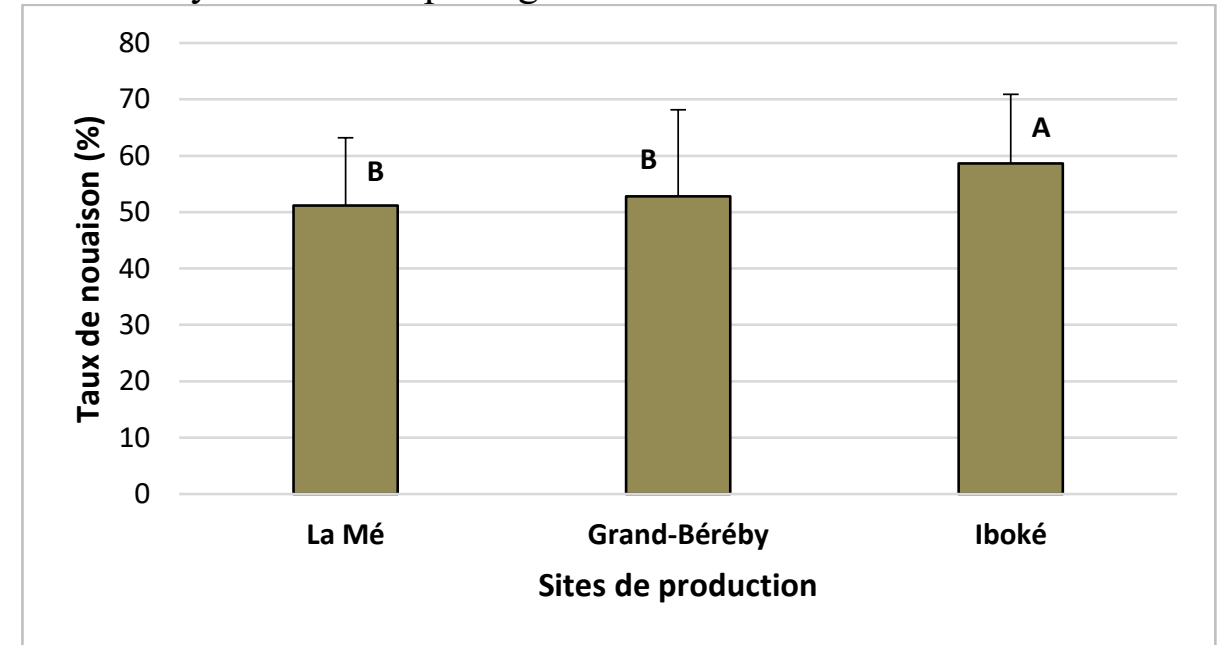

Figure 6 : Variation du taux de nouaison moyen des fruits sur les régimes en fonction des sites

Les barres représentent les écarts types des moyennes. Analyse de variance $: \mathrm{n}=2016, \mathrm{ddl}=215, F=6,21, \mathrm{p}=0,002$. Les histogrammes portant les mêmes lettres représentent des moyennes qui ne diffèrent pas significativement au seuil de $5 \%$.

\section{Incidence des attaques de Prosaustus spp sur le taux de nouaison}

Sur le site de La Mé, le taux de nouaison est corrélé négativement avec le pourcentage de fruits attaqués par l'espèce $P$. sculptilis $(\mathrm{r}=-0,45 ; \mathrm{p}<0,01)$, de même qu'avec le pourcentage total de fruits attaqués par les deux espèces de Prosœustus $(\mathrm{r}=-0,43 ; \mathrm{p}<0,01)$ (Tableau 5). 
A Grand-Béréby également, le taux de nouaison est corrélé négativement avec le pourcentage de fruits attaqués par P. sculptilis $(\mathrm{r}=-0,38$ $; \mathrm{p}<0,01)$, ainsi qu'avec le pourcentage total de fruits attaqués par les deux espèces $(\mathrm{r}=-0,42 ; \mathrm{p}<0,01)$ (Tableau 5$)$.

A Iboké par contre, aucune corrélation significative n'a été observée entre le taux de nouaison et les attaques des ravageurs.

Tableau 5 : Coefficients de corrélation entre le taux de nouaison et le pourcentage de fruits attaqués par les espèces de Prosœustus à La Mé, Grand-Béréby et Iboké

\begin{tabular}{lllll} 
& \multicolumn{3}{l}{ Variables indépendantes } \\
\hline \multirow{2}{*}{ Sites } & $\begin{array}{l}\text { Variable } \\
\text { dépendante }\end{array}$ & $\begin{array}{l}\text { \% fruits } \\
\text { attaqués par } \\
\text { P. minor }\end{array}$ & $\begin{array}{l}\text { \% fruits } \\
\text { attaqués par } \\
\text { P. sculptilis }\end{array}$ & $\begin{array}{l}\text { Total fruits } \\
\text { attaqués par les } \\
\text { deux espèces de } \\
\text { Prosæustus }\end{array}$ \\
\hline Moyenne La Mé & TN (\%) & $-0,05$ & $-0,45^{* *}$ & $-0,43^{* *}$ \\
Moyenne Grand- & TN (\%) & $-0,23$ & $-0,38^{* *}$ & $-0,42^{* *}$ \\
Béréby & MN $(\%)$ & $-0,12$ & $-0,11$ & $-0,14$ \\
\hline
\end{tabular}

TN : Taux de nouaison; $* \mathrm{p}<0,05 ; * * \mathrm{p}<0,01 ; * * * \mathrm{p}<0,001$

\section{Influence de l'abondance des insectes pollinisateurs}

Sur le site de La Mé, une corrélation positive et significative a été observée entre le taux de nouaison et les densités des espèces $E$. kamerunicus $(\mathrm{r}=0,47 ; \mathrm{p}=0,030)$ et E. plagiatus $(\mathrm{r}=0,44 ; \mathrm{p}=0,028)$ sur les fleurs mâles. De même, le taux de nouaison est corrélé avec le nombre de E. kamerunicus et celui de E. plagiatus qui visitent les fleurs femelles (respectivement : $\mathrm{r}=$ 0,$60 ; p=0,002$ et $r=0,47 ; p=0,020$ ). Ainsi, les taux de nouaison ont été plus élevés entre octobre et janvier ; période au cours de laquelle l'activité de ces deux espèces sur les fleurs femelles est plus importante (Figures 3 et 4).

Sur le site de Grand-Béréby, aucune corrélation significative n'a été observée entre le taux de nouaison moyen et la densité des différentes espèces d'insectes pollinisateurs collectés.

A Iboké enfin, le taux de nouaison est corrélé significativement avec la densité des espèces E. subvittatus $(\mathrm{r}=0,40 ; \mathrm{p}<0,001), M$. congolense $(\mathrm{r}=$ $0,41 ; \mathrm{p}=0,046), M$. dispar $(\mathrm{r}=0,57 ; \mathrm{p}=0,004)$ et A. burgeoni $(\mathrm{r}=0,65 ; \mathrm{p}$ $<0,001)$ sur les fleurs mâles. Ainsi la Figure 5 montre que la nouaison est meilleure lorsque la densité de ces espèces est plus élevée, entre décembre et juin. 


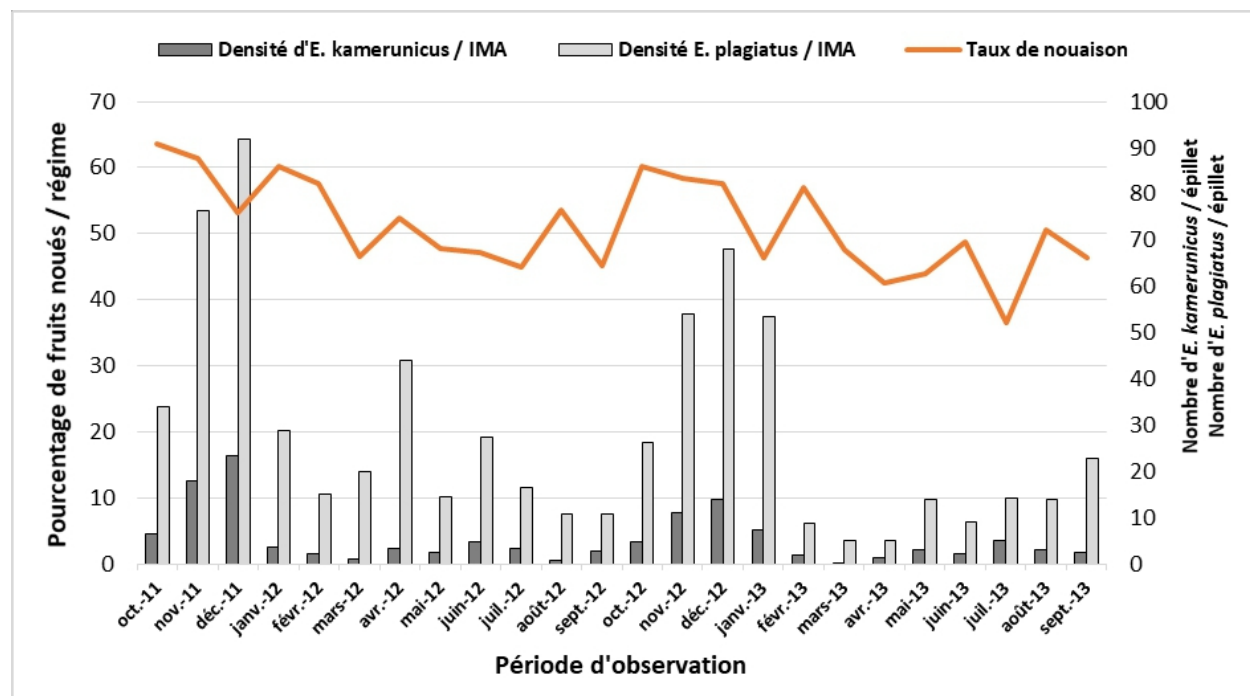

Figure 7 : Evolution du taux de nouaison en fonction de la densité d'E. kamerunicus et $E$. plagiatus sur les fleurs mâles à La Mé

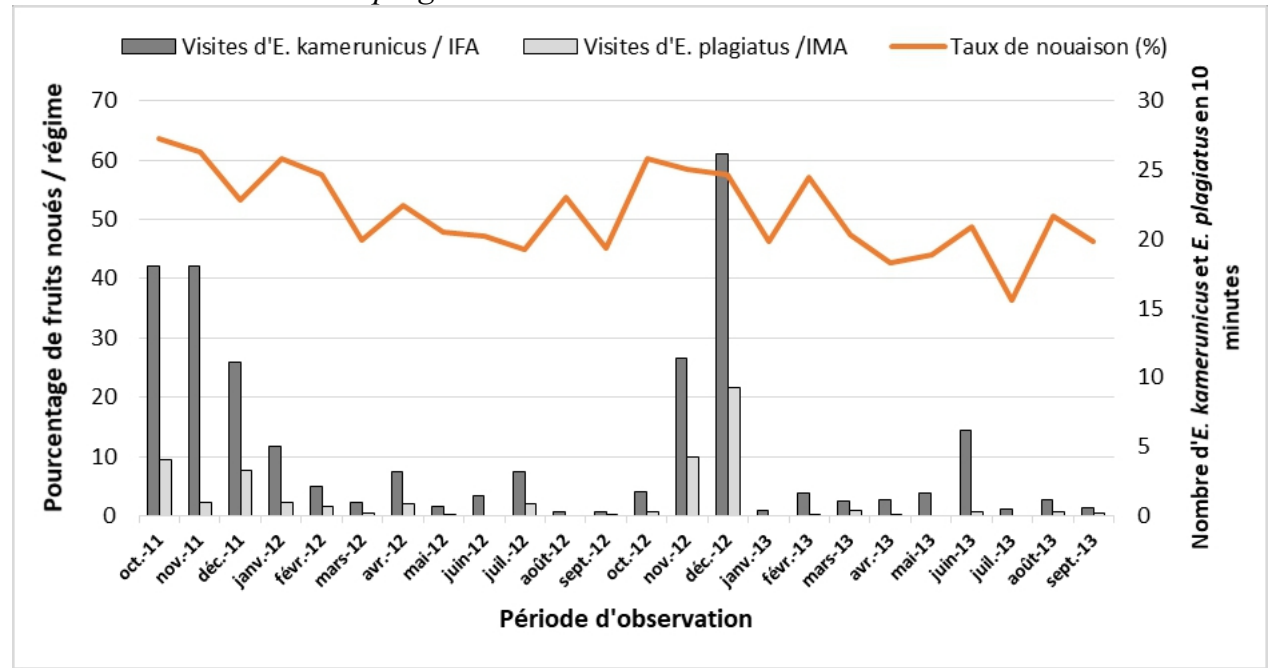

Figure 8 : Evolution du pourcentage de fruits noués en fonction du nombre d' $E$. kamerunicus et E. plagiatus visitant les fleurs femelles à La Mé 


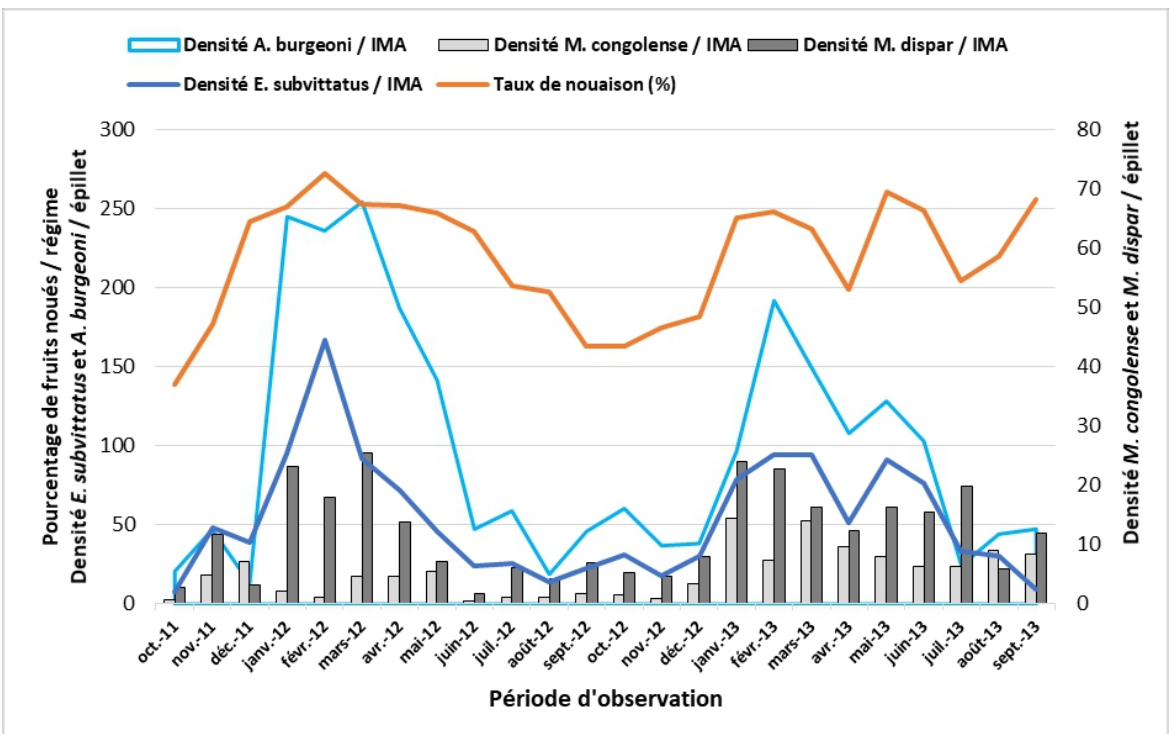

Figure 9 : Evolution du pourcentage de fruits noués en fonction de la densité d' $E$. subvittatus, $M$. congolense, $M$. dispar et $A$. burgeoni sur les fleurs mâles à Iboké

\section{Discussion}

Les collectes effectuées au cours des 24 mois ont montré que la composition de l'entomofaune des inflorescences, en termes de nombre d'espèces, est similaire sur les trois sites d'étude. En effet, toutes les espèces listées par Desmier de Chenon (1981) ont été retrouvées dans les différentes parcelles des trois sites. Il s'agit également des espèces recensées par Hala et al. (2012) sur la station de La Mé.

Parmi les insectes rencontrés sur les inflorescences mâles, certains ne semblent pas fréquenter les inflorescences femelles. Desmier de Chenon (1981) affirmait en effet que parmi la vingtaine d'espèces rencontrées sur les fleurs mâles, seul un petit nombre fréquente les fleurs femelles. Les espèces du genre Elaeidobius ont été les plus rencontrées simultanément sur les deux types de fleurs. Ce résultat confirme le rôle prépondérant joué par ces insectes dans la pollinisation du palmier à huile (Appiah et Agyei-Dwarko, 2013; Syarifah et Idris, 2016). Ces espèces vivent sur les inflorescences mâles au niveau desquelles elles se nourrissent et se reproduisent. Mais, lorsque les inflorescences femelles sont en anthèse, elles produisent le même parfum que les mâles. C'est ce parfum qui les attire (Jacquemard, 1995). Les espèces de Microporum et l'espèce Atheta burgeoni ont aussi été retrouvées sur les deux types d'inflorescences. Toutefois, malgré la forte abondance de Atheta burgeoni sur les inflorescences mâles, elle fréquente moins les inflorescences femelles que les espèces d'Elaeidobius.

Les espèces de Prosœustus (P. minor et $P$. sculptilis) ont été également rencontrées sur les deux types d'inflorescences, mais elles sont plus 
abondantes sur les fleurs femelles. En effet, contrairement aux espèces d'Elaeidobius qui se reproduisent sur les inflorescences mâles, les adultes de Prosœustus pondent leurs œufs sur les fleurs femelles au niveau desquelles les larves se nourrissent en faisant des dégâts (Philippe, 1993). Cela justifie donc leur plus grande présence sur les inflorescences femelles.

Les populations des deux espèces A. burgeoni et E. subvittatus dominent fortement celles des autres insectes. L'espèce $E$. kamerunicus a été faiblement abondante dans les plantations. Ces résultats sont conformes à ceux obtenus par Hala et al. (2012) sur le site de La Mé. Pourtant, dans les régions de l'Asie du Sud-ouest où E. kamerunicus fut introduit dans les années 1980, cet insecte s'est établi en dominant l'espèce native E. subvittatus. Ces densités peuvent atteindre plus de 50 E. kamerunicus par épillet (Syarifah et Idris, 2016). Labarca et al. (2009) ont obtenu des proportions supérieures à $90 \%$ dans certaines plantations du Venezuela, contre environ $4 \%$ pour E. subvittatus.

Les faibles densités de l'espèce E. kamerunicus pourraient être causées par un ensemble de facteurs. En effet, cette espèce possède des ennemis naturels tels que les nématodes parasites Elaeolenchus parthenonema n. g., n. sp. et Cylindrocorpus invectus sp. n., (Poinar et al., 2002 ; Poinar et al., 2003). Certains des insectes qui fréquentent les inflorescences pourraient également être des prédateurs ou des parasites de E. kamerunicus. En Côte d'Ivoire, l'inventaire des ennemis naturels de l'insecte n'a pas encore été faite.

L'étude a déterminé des taux de nouaison d'environ $51 \%$ à La Mé, 52 $\%$ à Grand-Béréby et $58 \%$ à Iboké. Le taux de nouaison sur 1'ensemble des sites peut donc être qualifié de moyen. Ce niveau de nouaison pourrait être lié à la qualité de la pollinisation. En effet, l'espèce E. kamerunicus qui possède un pouvoir pollinisateur supérieur (Appiah et Agyei-Dwarko, 2013 ; Kouakou et al., 2014) a été moins abondante sus les inflorescences, au profit de $A$. burgeoni et E. subvittatus. Des observations antérieurs ont montré que le taux de nouaison est meilleur lorsque les espèces comme E. kamerunicus et $E$. plagiatus sont abondantes sur les inflorescences. Au contraire, lorsque l'entomofaune est dominée par E. subvittatus, la nouaison est moins bonne (Mariau et Genty, 1988 ; Mariau et al., 1991).

A La Mé, Grand-Béréby et Iboké, les analyses ont également mis en évidence une influence négative et significative de dégâts causés par les ravageurs Prosœustus spp sur le taux de nouaison. Ces insectes ravageurs constituent donc la deuxième cause de diminution du taux de nouaison. A l'ouverture des spathes, les femelles de Prosœustus spp pondent des œufs dans de petites cavités creusées au niveau des stigmates. Dès l'éclosion, les larves creusent des galeries dans le style ou dans l'ovaire pour consommer la pulpe en formation, occasionnant un avortement ou une destruction des fruits. Ces dommages entrainent ainsi une diminution du taux de nouaison (Alibert, 1938 ; Philippe, 1993 ; Jacquemard, 2011). 


\section{Conclusion}

Le suivi simultané de l'entomofaune des inflorescences et du taux de nouaison dans les plantations a révélé une faible abondance de l'espèce $E$. kamerunicus, meilleur pollinisateur du palmier à huile. Cette faible densité et la présence d'insectes ravageurs sur les fleurs femelles constituent les principales causes de la diminution du taux de nouaison dans les différentes zones de production.

\section{Remerciements}

Ces travaux ont été financés par l'interprofession de la filière palmier à huile de Côte d'Ivoire (AIPH) à travers le Fonds Interprofessionnel pour la Recherche et le Conseil Agricole (FIRCA). Nous tenons à remercier ces structures.

\section{References:}

1. Agus, E.P., Wiharti, O.P. and Agus S., (2014). Elaeidobius kamerunicus: Application of hatch and carry technique for increasing oil palm fruit set. Journal of Oil Palm Research, 26 (3), 195-2012. http://jopr.mpob.gov.my/wpcontent/uploads/2014/10/joprv26sep2014-agus1.pdf (Site consulté le 20/03/2018).

2. Alibert, H., (1938). Etude sur les insectes parasites du palmier à huile au Dahomey. Journal d'agriculture traditionnelle et de Botanique Appliquée 207 : 745-773. http://www.persee.fr/doc/jatba 03703681 1938 num $18 \quad 207 \quad 5916$ (Site consulté le 20/03/2018).

3. Appiah, S.O., and Agyei-Dwarko, D., (2013). Studies on Entomophil pollination towards sustainable production and increased profitability in the oil Palm: a review. Elixir Agriculture, 55 (2013), 12878-12883. http://www.elixirpublishers.com/articles/1361001902 55\%20(2013) \%2012878-12883.pdf (Site consulté le 20/03/2018).

4. Appiah, S.O., (1999). Studies into entomophil pollination of Oil palm. Journal of the Ghana Science Association, 1(2), 70-77.

5. http://dx.doi.org/10.4314/jgsa.v1i2.17807

6. Brou, T.Y., (2010). Variabilité climatique, déforestation et dynamique agro démographique en Côte d'Ivoire. Sécheresse, 21 (1), 1-6.

7. http://dx.doi.org/10.1684/sec.2010.0277

8. Desmier de Chenon, R., (1981). Entomophil pollination of oil palm in West Africa. Preliminary research. In: the oil palm in agriculture in the eighties. Incorporated Society of Planters Ed., Malaysia, 1 (1), 231291.

9. Hala, N., Tuo, Y., Akpesse, A.A.M., Koua, H.K. et Tano, Y., (2012). Entomofauna of Oil palm tree inflorescences at La Mé Experimental 
Station. American Journal of Experimental Agriculture, 2(3), 306-319. http://dx.doi.org/10.9734/AJEA/2012/1128

10. Jacquemard, J.C., (1995). Le palmier à huile. Maison neuve et Larose ed, Paris France, 33p.

11. Jacquemard, J.C., (2011). Le palmier à huile. Agriculture Tropicale en Poche. Edition Quæ, CTA, Presses agronomiques de Gembloux. 275p.

12. Jannot, C., 2010. Emplois, économie, environnement : le développement de la filière palmier à huile en Côte d'Ivoire. $O C L, 17$ (6), 393-399. http://dx.doi.org/10.1051/ocl.2010.0344

13. Jianjun, Y., Zhen, Y., Cheng, B., Zetan, C., Weifu, L., and Fangzhen, J., (2015). Pollination Activity of Elaeidobius kamerunicus (Coleoptera: Curculionoidea) on Oil Palm on Hainan Island. Florida Entomologist 98 (2), 499-505. https://doi.org/10.1653/024.098.0217

14. Koua, K.H., Akpesse, A.K.M., Tuo, Y., and Hala N., (2013). Influence of the growing area on oil palm (Elaeis guineensis) inflorescences insects population. Journal of Research in Biology, 3(4), 967-976. http://jresearchbiology.com/documents/RA0344.pdf.

15. Kouakou, M., Hala, N., Akpesse, A.K.M., Tuo, Y., Dagnogo, M., Konan, K.E. and Koua, K.H., (2014). Comparative efficacy of Elaeidobius kamerunicus, E. Plagiatus, E. Subvittatus (Coleoptera: Curculionidae) and Microporum spp (Coleoptera: Nitidulidae) in the pollination of oil palm (Elaeis guineensis Jacq.). Journal of Experimental Biology and Agricultural Sciences, 2 (6), 538-545.

16. http://jebas.org/00200620122014/Kouakou\%20et\%20al\%20JEBAS.p df

17. Labarca, M.V., Portillo, E., Portillo, A. and Morales, E., (2009). Reproductive structures and the oil palm (Elaeis guineensis Jacq.) pollination by insects in three commercial fields in Zulia State, Venezuela. Rev. Fac. Agron. (LUZ), 26, 1-22.

18. Lecoustre, R., et De Reffey, P., (1987). Méthode d'estimation de la part due à la pollinisation dans l'expression du taux de nouaison.

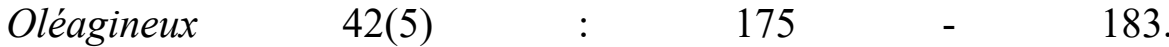
https://agritrop.cirad.fr/459059/1/ID459059.pdf (Site visité le 20/03/2018).

19. Mariau, D., et Genty, P., (1988). Contribution de l'IRHO à l'étude des insectes pollinisateurs du palmier à huile en Afrique, Amérique du Sud et Indonésie. Oléagineux 43(6), 233-240.

20. https://agritrop.cirad.fr/438175/1/ID438175.pdf $\quad$ (visité le 20/03/2018).

21. Mariau, D., Houssou, M., Lecoustre, R., and Ndigui, B., (1991). Insectes pollinisateurs du palmier à huile et taux de nouaison en Afrique de l'ouest. Oléagineux, 46(2), 43-51. 
22. https://agritrop.cirad.fr/427287/1/ID427287.pdf

23. Philippe, R., (1993). Etude de l'incidence des ravageurs sur les inflorescences femelles du palmier à huile en Afrique de l'Ouest.

Oléagineux, $48(10)$,

389 -

403.http://agritrop.cirad.fr/395607/1/ID395607.pdf （Site visité le 20/03/2018).

24. Poinar, G.O., Jackson, T.A., Bell, N.L., and Wahid, M.B., (2003). Cylindrocorpus invectus $s p$. n. associated with oil palm weevil Elaeidobius kamerunicus Faust (Coleoptera: Curculionidae), with synopsis of the family cylindrocorporidae and establishment of Longibuccidae. Nematology, 5: 183-190.

25. Poinar, G.O., Jackson, T.A., Belle, N.L., and Wahid, M.B., (2002). Elaeolenchus parthenonema n. g., n. sp. (Nematoda: Sphaerularioidea: Anandranematidae n. fam.) parasitic in the palm-pollinating weevil Elaeidobius kamerunicus Faust, with a phylogenetic synopsis of the Sphaerularioidea Lubbock. Syst. Parasitol., 52, 219-225.

26. Syarifah, N.S.M.D., and Idris, A.G., (2016). Population density of oil palm pollinator weevil Elaeidobius kamerunicus based on seasonal effect and age of oil palm. AIP Conference Proceedings 1784, 060051 (2016). http://dx.doi.org/10.1063/1.4966889.

27. Syed, R.A., (1979). Studies on oil palm pollination by insects. Bulletin of Entomological Research 69: 213-224. https://doi.org/10.1017/S0007485300017673

28. Syed, R.A., Law, I.H., and Corley, R.H.V., (1982). Insect pollination of Oil palm: Introduction, establishment and pollinating efficiency of Elaeidobius kamerunicus in Malaysia. Planter, 58(681), 547-561. https://www.cabdirect.org/cabdirect/abstract/19830312209

29. Tuo, Y., 2013. Etat de l'entomofaune des inflorescences du palmier à huile en Côte d'Ivoire : cas de la station CNRA de la Mé. Thèse es sciences. Université Félix Houphouët-Boigny, Abidjan, Côte d'Ivoire, $266 \mathrm{p}$. 